\title{
Uniqueness and partial identification in a geometric inverse problem for the Boussinesq system
}

\author{
Anna Doubova $^{a}$, Enrique Fernández-Cara ${ }^{\mathrm{a}}$, Manuel González-Burgos ${ }^{\mathrm{a}}$, Jaime Ortega b,c \\ ${ }^{a}$ Dpto. E.D.A.N., Universidad de Sevilla, Aptdo. 1160, 41080 Sevilla, Spain \\ ${ }^{\mathrm{b}}$ Departamento de Ciencias Básicas, Universidad del Bí-Bío, Casilla 447, Campus Fernando May, Chillán, Chile \\ ${ }^{\mathrm{c}}$ Centro de Modelamiento Matemático, Universidad de Chile, Casilla 170/3 Correo 3, Santiago, Chile \\ Received $* * * * * ;$ accepted after revision +++++ \\ Presented by
}

\begin{abstract}
We analyze the inverse problem of the identification of a rigid body immersed in a fluid governed by the stationary Boussinesq system. First, we establish a uniqueness result. Then, we present a new method for the partial identification of the body. The proofs use local Carleman estimates, differentiation with respect to domains, data assimilation techniques and controllability results for PDEs. To cite this article: A. Doubova, E. Fernández-Cara, M. González-Burgos, J.H. Ortega, C. R. Acad. Sci. Paris, Ser. I 340 (2005).
\end{abstract}

\section{Résumé}

Sur l'unicité et l'identification partielle d'un problème inverse géométrique pour le système de Boussinesq. On analyse le problème inverse de l'identification d'un corps rigide dans un fluide régi par le système stationnaire de Boussinesq. On établit d'abord un résultat d'unicité. Ensuite on présente une nouvelle méthode pour l'identification partielle du corps. Les preuves utilisent des estimations locales de Carleman, la différentiation par rapport au domaine, des techniques d'assimilation de données et des résultats de contrôlabilité des EDPs. Pour citer cet article : A. Doubova, E. Fernández-Cara, M. González-Burgos, J.H. Ortega, C. R. Acad. Sci. Paris, Ser. I 340 (2005).

\section{Version française abrégée}

Soient $\Omega \subset \mathbb{R}^{N}(N=2,3)$ un ouvert borné simplement connexe de frontière $\partial \Omega$ de classe $W^{2, \infty}$ et $\gamma \subset \partial \Omega$ un ouvert relatif non vide. On considère la famille $\mathcal{D}$ des sous-ensembles de $\Omega$ donnée par (3).

Email addresses: doubova@us.es (Anna Doubova), cara@us.es (Enrique Fernández-Cara), manoloburgos@us.es (Manuel González-Burgos), jortega@dim.uchile.cl (Jaime Ortega). 
On analyse le problème inverse suivant : Etant donnés $(\varphi, \psi)$ et $(\alpha, \beta)$ dans des espaces appropriés, on cherche un ensemble $D \in \mathcal{D}$ tel qu'une solution $(u, p, \theta)$ du système de Boussinesq

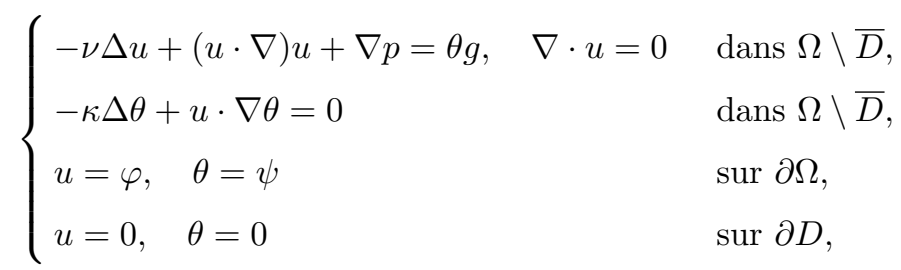

satisfait les conditions additionnelles

$$
\sigma(u, p) \cdot n:=(-p \operatorname{Id} .+2 \nu e(u)) \cdot n=\alpha, \quad \kappa \frac{\partial \theta}{\partial n}=\beta \quad \text { sur } \quad \gamma .
$$

Ici, $u, p$ et $\theta$ sont respectivement la vitesse, la pression et la température du fluide, $g$ est la force de la gravité, $\nu$ et $\kappa$ sont des constantes positives, Id. est la matrice identité et $e(u)=\frac{1}{2}\left(\nabla u+{ }^{t} \nabla u\right)$.

Un problème similaire pour l'équation de Laplace a été analysé dans [5]; un autre problème similaire pour le système de Stokes est considéré dans [1] ; finalement, le cas du système de Navier-Stokes est traité dans [3].

En ce qui concerne le problème direct, étant donnés $D \in \mathcal{D}$ et $(\varphi, \psi) \in H^{3 / 2}(\partial \Omega)^{N} \times H^{3 / 2}(\partial \Omega)$ avec $\int_{\partial \Omega} \varphi \cdot n d \Gamma=0$, il existe au moins une solution $(u, p, \theta)$ de (1) qui appartient à $H^{2}(\Omega \backslash \bar{D})^{N} \times H^{1}(\Omega \backslash$ $\bar{D}) \times H^{2}(\Omega \backslash \bar{D})$. De plus, si $(\varphi, \psi)$ est suffisamment petit (ce qui sera supposé par la suite), cette solution est unique ( $p$ est unique à une constante près). Le premier résultat de cette note concerne l'unicité :

Théorème 0.1 On suppose que $(\varphi, \psi) \neq(0,0)$. Soient $D^{i} \in \mathcal{D},\left(u^{i}, p^{i}, \theta^{i}\right)$ la solution de (1) avec $D$ remplacé par $D^{i}, \alpha^{i}=\sigma\left(u^{i}, p^{i}\right) \cdot n$ et $\beta^{i}=\kappa \frac{\partial \theta^{i}}{\partial n}$ pour $i=0,1$. Si $\left(\alpha^{0}, \beta^{0}\right)=\left(\alpha^{1}, \beta^{1}\right)$ sur $\gamma$, alors $D^{0}=D^{1}$.

Pour la preuve, il suffit d'adapter un argument introduit dans [5] et déjà utilisé dans [1] et [3]. On aura besoin d'une propriété de continuation unique appropriée, qu'on peut déduire en utilisant les idées de [4].

Pour l'identification partielle de $D$, on introduit la famille

$$
\mathcal{W}=\left\{m \in W^{2, \infty}\left(\mathbb{R}^{N} ; \mathbb{R}^{N}\right):\|m\|_{W^{2, \infty}} \leq \varepsilon, m=0 \text { dans } \Omega \backslash D^{*}\right\},
$$

où $\varepsilon>0$ est suffisament petit. On pose

$$
D+m=\left\{z \in \mathbb{R}^{N}: z=x+m(x), x \in D\right\}
$$

pour tout $m \in \mathcal{W}$. On considère maintenant, le système de Boussinesq "perturbé" (6). La question de l'identification partielle de $D$ est la suivante : on suppose $D \in \mathcal{D}$ connu, donc on peut résoudre le problème direct (1) et calculer $(\alpha, \beta)$ à partir de (2); aussi, on suppose que l'observation $\left(\alpha^{m}, \beta^{m}\right)$ associée à $D+m$ est connue; alors on veut calculer $\left.m \cdot n\right|_{\partial D}$ à partir de $D,(\alpha, \beta)$ et $\left(\alpha^{m}, \beta^{m}\right)$.

Le deuxième résultat de cette note est le suivant :

Théorème 0.2 Soient $(\varphi, \psi) \in H^{3 / 2}(\partial \Omega)^{N} \times H^{3 / 2}(\partial \Omega),(\varphi, \psi) \not \equiv(0,0)$ avec $\int_{\partial \Omega} \varphi \cdot n d \Gamma=0$ et la solution correspondante de (1) vérifie $\left|\frac{\partial u}{\partial n}\right|^{2}+\left|\frac{\partial \theta}{\partial n}\right|^{2} \neq 0$ sur $\partial D$. On suppose que $m \in \mathcal{W}$ et $\left.(m \cdot n)\right|_{\partial D} \in M$ où $M$ est un sous-espace de dimension fini de $W^{1, \infty}(\partial D)$. Alors $\left.(m \cdot n)\right|_{\partial D}$ peut être calculé explicitement, à des perturbations de deuxième ordre près, à partir de $\Omega, D, M,(\alpha, \beta)$ et $\left(\alpha^{m}, \beta^{m}\right)$. Plus précisement, il existe une application linéaire calculable $H_{\Omega, D, M}: H^{1 / 2}(\gamma)^{N} \times H^{1 / 2}(\gamma) \mapsto M$ telle que

$$
\left.(m \cdot n)\right|_{\partial D}=H_{\Omega, D, M}\left(\alpha^{m}-\alpha, \beta^{m}-\beta\right)+o(m)
$$

pour tout $m \in \mathcal{W}$ avec $\left.(m \cdot n)\right|_{\partial D} \in M$, où o $(m)\|m\|_{W^{2}, \infty}^{-1} \rightarrow 0$ quand $\|m\|_{W^{2, \infty}} \rightarrow 0$.

Pour la preuve, on utilise la méthode de variation des domaines introduite dans [6] et des techniques d'assimilation des données introduites dans [7]. Les démonstrations détaillées de deux théorèmes précédents seront données dans un article à paraître. 


\section{Introduction}

Let $\Omega \subset \mathbb{R}^{N}(N=2,3)$ be a simply connected bounded open set with boundary $\partial \Omega$ of class $W^{2, \infty}$ and let $n$ be the unit outward normal vector to $\Omega$. Let $\gamma \subset \partial \Omega$ be a nonempty relative open subset and let us denote by $1_{\gamma}$ the characteristic function of $\gamma$. Let $D^{*}$ be a fixed nonempty open set such that $D^{*} \subset \subset \Omega$ and let us consider the following family of subsets of $\Omega$ :

$\mathcal{D}=\left\{D \subset \Omega: D\right.$ is a simply connected nonempty open set, $\partial D$ is of class $\left.W^{2, \infty}, D \subset \subset D^{*}\right\}$.

In this note, we will be concerned with the following inverse problem: Given $(\varphi, \psi)$ and $(\alpha, \beta)$ in appropriate spaces, find a set $D \in \mathcal{D}$ such that a solution $(u, p, \theta)$ of the Boussinesq system

$$
\begin{cases}-\nu \Delta u+(u \cdot \nabla) u+\nabla p=\theta g, & \nabla \cdot u=0 \\ -\kappa \Delta \theta+u \cdot \nabla \theta=0 & \text { in } \Omega \backslash \bar{D}, \\ u=\varphi, \quad \theta=\psi & \text { in } \Omega \backslash \bar{D}, \\ u=0, \quad \theta=0 & \text { on } \partial \Omega,\end{cases}
$$

satisfies the additional conditions

$$
\sigma(u, p) \cdot n:=(-p \operatorname{Id} .+2 \nu e(u)) \cdot n=\alpha, \quad \kappa \frac{\partial \theta}{\partial n}=\beta \quad \text { on } \quad \gamma \cdot
$$

In (4), $u, p$ and $\theta$ are respectively the velocity, the pressure and the temperature of the fluid, $g$ is the gravitational force, $\nu>0$ is the kinematic viscosity and $\kappa>0$ is the thermal conductivity. In (5), Id. is the identity matrix and $e(u)=\frac{1}{2}\left(\nabla u+{ }^{t} \nabla u\right)$ is the linear strain tensor.

A similar problem has been analyzed in [5]. A related problem concerning a Stokes fluid was considered in [1]; see also [3] for the analysis of a similar problem for the Navier-Stokes system. Concerning the direct problem, given $D \in \mathcal{D}$ and $(\varphi, \psi) \in H^{3 / 2}(\partial \Omega)^{N} \times H^{3 / 2}(\partial \Omega)$ with $\int_{\partial \Omega} \varphi \cdot n d \Gamma=0$, there exists at least one solution $(u, p, \theta)$ of (4) that belongs to $H^{2}(\Omega \backslash \bar{D})^{N} \times H^{1}(\Omega \backslash \bar{D}) \times H^{2}(\Omega \backslash \bar{D})$. Moreover, if $(\varphi, \psi)$ is sufficiently small (and this will be assumed in the sequel), then the solution of (4) is unique ( $p$ is unique up to a constant). Our first result in this note concerns uniqueness and is the following:

Theorem 1.1 Assume that $(\varphi, \psi) \neq(0,0)$. Let $D^{i} \in \mathcal{D},\left(u^{i}, p^{i}, \theta^{i}\right)$ be the solution of (4) with $D$ replaced by $D^{i}$ and set $\alpha^{i}=\sigma\left(u^{i}, p^{i}\right) \cdot n$ and $\beta^{i}=\kappa \frac{\partial \theta^{i}}{\partial n}$ for $i=0,1$. If $\left(\alpha^{0}, \beta^{0}\right)=\left(\alpha^{1}, \beta^{1}\right)$ on $\gamma$, then $D^{0}=D^{1}$.

For the proof, we adapt an argument introduced in [5] and already used in [1] and [3]. To this end, we need an appropriate unique continuation property, that can be obtained using the ideas of [4].

For the partial identification of $D$, let us introduce the family

$$
\mathcal{W}=\left\{m \in W^{2, \infty}\left(\mathbb{R}^{N} ; \mathbb{R}^{N}\right):\|m\|_{W^{2, \infty}} \leq \varepsilon, \quad m=0 \text { in } \Omega \backslash D^{*}\right\},
$$

where $\varepsilon>0$ is sufficiently small and let us set

$$
D+m=\left\{z \in \mathbb{R}^{N}: z=x+m(x), x \in D\right\}
$$

for all $m \in \mathcal{W}$. Notice that, for any $D \in \mathcal{D}$ and any $m \in \mathcal{W}$, one has again $D+m \in \mathcal{D}$; see for instance [8]. For each $m \in \mathcal{W}$, let us consider the "perturbed" Boussinesq system

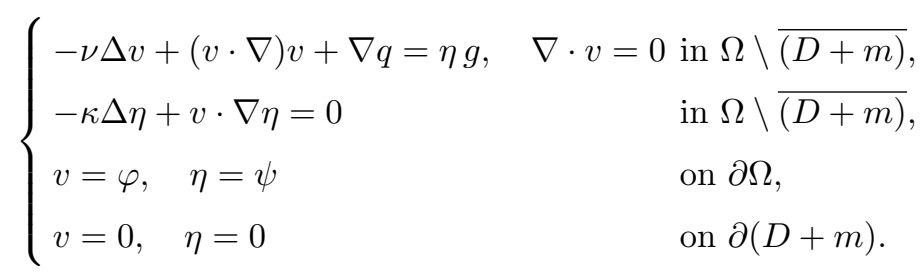


Let us set $\left(\alpha^{m}, \beta^{m}\right)=\left(\left.\sigma(v, q) \cdot n\right|_{\gamma},\left.\kappa \frac{\partial \eta}{\partial n}\right|_{\gamma}\right)$. The partial identification problem for $D$ is the following: we assume that $D \in \mathcal{D}$ is known, so we can solve the direct problem (4) and compute $(\alpha, \beta)$ from (5); we also assume that we know $\left(\alpha^{m}, \beta^{m}\right)$; then we want to compute $\left.m \cdot n\right|_{\partial D}$ from $D,(\alpha, \beta)$ and $\left(\alpha^{m}, \beta^{m}\right)$.

Our second result in this note is the following:

Theorem 1.2 Let $(\varphi, \psi) \in H^{3 / 2}(\partial \Omega)^{N} \times H^{3 / 2}(\partial \Omega),(\varphi, \psi) \not \equiv(0,0)$ with $\int_{\partial \Omega} \varphi \cdot n d \Gamma=0$ and the corresponding solution of (4) satisfies $\left|\frac{\partial u}{\partial n}\right|^{2}+\left|\frac{\partial \theta}{\partial n}\right|^{2} \neq 0$ on $\partial D$. Also, assume that $m \in \mathcal{W}$ and $\left.(m \cdot n)\right|_{\partial D} \in$ $M$, where $M$ is a finite dimensional subspace of $W^{1, \infty}(\partial D)$. Then $\left.(m \cdot n)\right|_{\partial D}$ can be computed explicitly, up to second-order terms, from $\Omega, D, M,(\alpha, \beta)$ and $\left(\alpha^{m}, \beta^{m}\right)$. More precisely, there exists a computable linear mapping $H_{\Omega, D, M}: H^{1 / 2}(\gamma)^{N} \times H^{1 / 2}(\gamma) \mapsto M$ such that

$$
\left.(m \cdot n)\right|_{\partial D}=H_{\Omega, D, M}\left(\alpha^{m}-\alpha, \beta^{m}-\beta\right)+o(m)
$$

for all $m \in \mathcal{W}$ with $\left.(m \cdot n)\right|_{\partial D} \in M$, where $o(m)\|m\|_{W^{2, \infty}}^{-1} \rightarrow 0$ as $\|m\|_{W^{2, \infty}} \rightarrow 0$.

For the proof, we use some domain variation techniques introduced in [6] and also some recent results on data assimilation introduced in [7].

In the following sections, we will indicate the main ideas of the proofs of theorems 1.1 and 1.2. The detailed proofs will be given in a forthcoming paper.

\section{Proof of the uniqueness result}

The main ingredient in the proof of Theorem 1.1 is the following unique continuation property:

Proposition 2.1 Let $G \subset \mathbb{R}^{N}$ be a bounded connected open set with boundary $\partial G$ of class $W^{1, \infty}$. Let $\Gamma \subset \partial G$ be a nonempty open set. Assume that $a \in L^{\infty}(G)^{N}, b \in L^{\infty}(G)^{N}, d \in L^{\infty}(G)$ and $\nabla \cdot a=\nabla \cdot b=0$ in $G$. Then any solution $(y, \lambda, \zeta) \in H^{1}(G)^{N} \times L^{2}(G) \times H^{1}(G)$ of

$$
\left\{\begin{array}{lr}
-\nu \Delta y+(a \cdot \nabla) y+(y \cdot \nabla) b+\nabla \lambda=\zeta g, \quad \nabla \cdot y=0 & \text { in } G, \\
-\kappa \Delta \zeta+a \cdot \nabla \zeta+y \cdot \nabla d=0 & \text { in } G,
\end{array}\right.
$$

that satisfies $y=0, \zeta=0$ on $\Gamma$ and $\sigma(y, \lambda) \cdot n=0, \kappa \frac{\partial \zeta}{\partial n}=0$ on $\Gamma$ is zero everywhere.

For the proof of this result we use some local Carleman inequalities obtained using the arguments of [4].

Let us now give a sketch of the proof of theorem 1.1. We reproduce the argument introduced in [5]. We consider the open sets $D^{0} \cup D^{1}$ and $\mathcal{O}^{0}=\Omega \backslash \overline{D^{0} \cup D^{1}}$. Let $\mathcal{O}$ be the unique connected component of $\mathcal{O}^{0}$ such that $\partial \mathcal{O}=\partial \Omega$ and let us introduce

$$
w=u^{0}-u^{1}, \quad \chi=\theta^{0}-\theta^{1} \text { and } \pi=p^{0}-p^{1} \text { in } \mathcal{O},
$$

where $\left(u^{i}, p^{i}, \theta^{i}\right)$ is the solution of (4) with $D$ replaced by $D^{i}, i=0,1$. Then $(w, \pi, \chi) \in H^{1}(\mathcal{O})^{N} \times L^{2}(\mathcal{O}) \times$ $H^{1}(\mathcal{O})$ verifies $(7)$ in $\mathcal{O}$ for some $a, b$, and $d$ satisfying the assumptions in proposition 2.1. Furthermore, $w=0, \chi=0$ on $\partial \Omega$ and $\sigma(w, \pi) \cdot n=0, \kappa \frac{\partial \chi}{\partial n}=0$ on $\gamma$. Thanks to proposition 2.1 we deduce that $w=0$ and $\chi=0$ in $\mathcal{O}$, that is to say,

$$
u^{0}=u^{1} \quad \text { and } \quad \theta^{0}=\theta^{1} \quad \text { in } \quad \mathcal{O} .
$$

We assume for instance that $D^{1} \backslash \overline{D^{0}} \neq \emptyset$ and let us introduce the open set $D^{2}=D^{1} \cup\left(\left(\Omega \backslash \overline{D^{0}}\right) \cap(\Omega \backslash \overline{\mathcal{O}})\right)$. By hypothesis, $D^{2} \backslash \bar{D}^{0}$ is nonempty. Moreover, $\partial\left(D^{2} \backslash \overline{D^{0}}\right)=\Gamma^{0} \cup \Gamma^{1}$, where $\Gamma^{0}=\partial\left(D^{2} \backslash \overline{D^{0}}\right) \cap \partial D^{0}$ and $\Gamma^{1}=\partial\left(D^{2} \backslash \overline{D^{0}}\right) \cap \partial D^{1}$. Thus, $\left(u^{0}, p^{0}, \theta^{0}\right)$ satisfies $(7)$ in $D^{2} \backslash \overline{D^{0}}$ with $u^{0}=u^{1}=0, \theta^{0}=\theta^{1}=0$ on $\Gamma^{1}$ and $u^{0}=0, \theta^{0}=0$ on $\Gamma^{0}$. Therefore, $u^{0}=0$ in $D^{2} \backslash \overline{D^{0}}$ and $\theta^{0}=0$ in $D^{2} \backslash \overline{D^{0}}$. Consequently, from proposition 2.1 we deduce that $u^{0} \equiv 0$ in $\Omega \backslash \overline{D^{0}}$ and $\theta^{0} \equiv 0$ in $\Omega \backslash \overline{D^{0}}$, which is impossible because $u^{0}=\varphi$ on $\partial \Omega$ and $\theta^{0}=\psi$ on $\partial \Omega$ and $(\varphi, \psi)$ is not identically zero. This implies that $D^{1} \backslash \overline{D^{0}}$ is the empty set. We can prove in the same way that the set $D^{0} \backslash \overline{D^{1}}$ is empty. Therefore, $D^{0}=D^{1}$. 


\section{Sketch of the proof of the partial identification result}

Let us assume that $(\alpha, \beta)$ and $\left(\alpha^{m}, \beta^{m}\right)$ are known. Recall that the goal is to compute $\left.(m \cdot n)\right|_{\partial D} \in M$ explicitly (up to second-order terms) from $(\alpha, \beta)$ and $\left(\alpha^{m}, \beta^{m}\right)$.

Step 1 (Domain variations): Using domain variation techniques (cf. [8]) and the arguments in [2], we have the following identities:

$$
\left\{\begin{array}{l}
\alpha^{m}-\alpha \equiv \sigma(v, q) \cdot n-\sigma(u, p) \cdot n=\sigma\left(u^{\prime}, p^{\prime}\right) \cdot n+o(m) \text { on } \gamma \\
\beta^{m}-\beta \equiv \kappa \frac{\partial \eta}{\partial n}-\kappa \frac{\partial \theta}{\partial n}=\kappa \frac{\partial \theta^{\prime}}{\partial n}+o(m) \text { on } \gamma
\end{array}\right.
$$

where $m \in \mathcal{W}, o(m)\|m\|_{W^{2, \infty}}^{-1} \rightarrow 0$ as $\|m\|_{W^{2, \infty}} \rightarrow 0$ and $\left(u^{\prime}, p^{\prime}, \theta^{\prime}\right) \in H^{1}(\Omega \backslash \bar{D})^{N} \times L^{2}(\Omega \backslash \bar{D}) \times H^{1}(\Omega \backslash \bar{D})$ is the solution of the linear problem

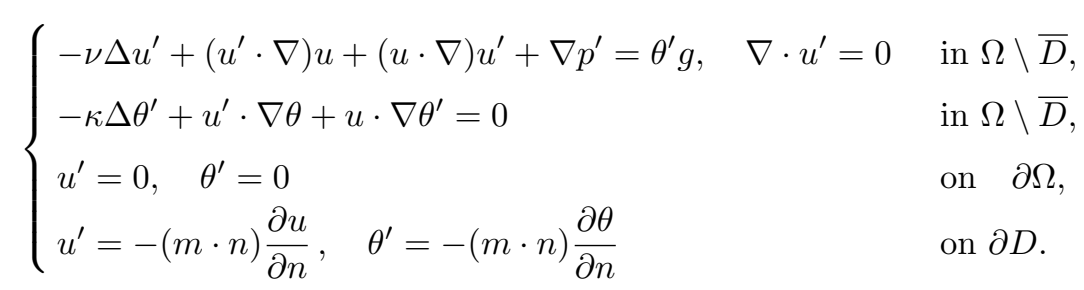

Thus, the proof is reduced to compute $\left.(m \cdot n)\right|_{\partial D}$ from $\left.\sigma\left(u^{\prime}, p^{\prime}\right) \cdot n\right|_{\gamma}$ and $\left.\kappa \frac{\partial \theta^{\prime}}{\partial n}\right|_{\gamma}$ up to second-order terms.

Step 2 (A non standard data assimilation approach): Let us now assume that $\left.(m \cdot n)\right|_{\partial D} \in M$. Then, in view of $(9)$, we also have $\left(\left.\sigma\left(u^{\prime}, p^{\prime}\right) \cdot n\right|_{\partial D},\left.\kappa \frac{\partial \theta^{\prime}}{\partial n}\right|_{\partial D}\right) \in E$, where $E \subset H^{-1 / 2}(\partial D)^{N} \times H^{-1 / 2}(\partial D)$ is an appropriate finite-dimensional space. Obviously, the quantities

$$
\int_{\partial D}(m \cdot n)\left(\left|\frac{\partial u}{\partial n}\right|^{2}+\left|\frac{\partial \theta}{\partial n}\right|^{2}\right) h d \Gamma, \quad h \in M
$$

determine $\left.(m \cdot n)\right|_{\partial D}$. So, our goal is to write these integrals in terms of $\left.\sigma\left(u^{\prime}, p^{\prime}\right) \cdot n\right|_{\gamma}$ and $\left.\kappa \frac{\partial \theta^{\prime}}{\partial n}\right|_{\gamma}$.

To this end, we will use an argument inspired by the data assimilation techniques introduced in [7]. Thus, let us assume for the moment that we can solve the following control problem: find a control $\left(w^{1}, w^{2}\right) \in H^{1 / 2}(\gamma)^{N} \times H^{1 / 2}(\gamma)$ such that the corresponding weak solution $(y, q, z) \in H^{1}(\Omega \backslash \bar{D})^{N} \times$ $L^{2}(\Omega \backslash \bar{D}) \times H^{1}(\Omega \backslash \bar{D})$ of

$$
\left\{\begin{array}{lc}
-\nu \Delta y-(\nabla y)^{t} u-(u \cdot \nabla) y+\nabla q=-z \nabla \theta, & \nabla \cdot y=0 \\
-\kappa \Delta z-u \cdot \nabla z=g \cdot y & \text { in } \Omega \backslash \bar{D} \\
y=w^{1} 1_{\gamma}, \quad z=w^{2} 1_{\gamma} \backslash \bar{D} \\
\sigma(y, q) \cdot n=\frac{\partial u}{\partial n} h, \quad \kappa \frac{\partial z}{\partial n}=\frac{\partial \theta}{\partial n} h & \text { on } \partial \Omega
\end{array}\right.
$$

satisfies

$$
\left\langle(\Phi, \Psi),\left(\left.y\right|_{\partial D},\left.z\right|_{\partial D}\right)\right\rangle_{\partial D}=0 \quad \forall(\Phi, \Psi) \in E .
$$

Then, taking into account (11) and that $\left(u^{\prime}, p^{\prime}, \theta^{\prime}\right)$ is the solution of $(9)$, we can write that

$$
-\int_{\partial D}(m \cdot n)\left(\left|\frac{\partial u}{\partial n}\right|^{2}+\left|\frac{\partial \theta}{\partial n}\right|^{2}\right) h d \Gamma=\left\langle\sigma(y, q) \cdot n, u^{\prime}\right\rangle_{\partial D \cup \partial \Omega}+\kappa\left\langle\frac{\partial z}{\partial n}, \theta^{\prime}\right\rangle_{\partial D \cup \partial \Omega}
$$




$$
=\left\langle\sigma\left(u^{\prime}, p^{\prime}\right) \cdot n, y\right\rangle_{\partial D \cup \partial \Omega}+\kappa\left\langle\frac{\partial \theta^{\prime}}{\partial n}, z\right\rangle_{\partial D \cup \partial \Omega}=\left\langle\sigma\left(u^{\prime}, p^{\prime}\right) \cdot n, w^{1} 1_{\gamma}\right\rangle_{\partial \Omega}+\kappa\left\langle\frac{\partial \theta^{\prime}}{\partial n}, w^{2} 1_{\gamma}\right\rangle_{\partial \Omega} .
$$

Therefore, we can compute $\left.(m \cdot n)\right|_{\partial D}$ (up to second-order perturbations) from $\left.\sigma\left(u^{\prime}, p^{\prime}\right) \cdot n\right|_{\gamma}$ and $\left.\kappa \frac{\partial \theta^{\prime}}{\partial n}\right|_{\gamma}$ and, as we have already seen in the previous step, from the known observations $\left(\alpha^{m}, \beta^{m}\right)$ and $(\alpha, \beta)$.

It remains only to justify that the previous control problem can be solved for all $h \in M$. But this is a consequence of the unique continuation property given in proposition 2.1 and the fact that $E$ has finite dimension. For the proof, we can follow (for instance) the method in [9]. This ends the proof of theorem 1.2.

Remark 1 From the practical viewpoint, what we have to do is the following. Let $\left\{\ell, \ldots, \ell_{I}\right\}$ be a basis of $M$ and let us put

$$
\left.(m \cdot n)\right|_{\partial D}=\sum_{i=1}^{I} a_{i} \ell_{i}
$$

Let $\left(w_{i}^{1}, w_{i}^{2}\right)$ be, for each $i=1, \ldots, I$ a control solving the problem (10)-(11) with $h=\ell_{i}$. Then the coefficients $a_{i}$ are given by the unique solution of the following linear system:

$$
\sum_{i=1}^{I}\left(\int_{\partial D} \ell_{i}\left(\left|\frac{\partial u}{\partial n}\right|^{2}+\left|\frac{\partial \theta}{\partial n}\right|^{2}\right) \ell_{j} d \Gamma\right) a_{i}=q_{j}, \quad 1 \leq j \leq I,
$$

where we have set $q_{j}=-\left\langle\sigma\left(u^{\prime}, p^{\prime}\right) \cdot n, w_{j}^{1} 1_{\gamma}\right\rangle_{\partial \Omega}-\kappa\left\langle\frac{\partial \theta^{\prime}}{\partial n}, w_{j}^{2} 1_{\gamma}\right\rangle_{\partial \Omega}$.

\section{Acknowledgements}

The first three authors were partially supported by D.G.E.S., grant BFM2003-06446 and the fourth author was partially supported by grant FONDECYT-CONICYT 1030943.

\section{References}

[1] C. Alvarez, C. Conca, L. Friz, O. Kavian, J.H. Ortega, An inverse problem for the Stokes system, to appear.

[2] J.A. Bello, E. Fernández-Cara, J. Lemoine, J. Simon, The differentiability of the drag with respect to the variations of a Lipschitz domain in a Navier-Stokes flow, SIAM J. Control Optim. 35 (2) (1997) 626-640.

[3] A. Doubova, E. Fernández-Cara, J.H. Ortega, A geometric inverse problem for the Navier-Stokes equation, to appear.

[4] C. Fabre, G. Lebeau, Prolongement unique des solutions de l'équation de Stokes, Comm. PDE 21 (1996) 573-596.

[5] O. Kavian, Four lectures on parameter identification in elliptic partial differential operators, Lectures at the University of Sevilla, Spain, 2002.

[6] F. Murat, J. Simon, Quelques résultats sur le contrôle par un domaine géométrique, Rapport du L.A. 189 no. 74003 , Université Paris VI, 1974.

[7] J.P. Puel, A nonstandard approach to a data assimilation problem, C. R. Math. Acad. Sci. Paris 335 no. 2 (2002) $161-166$.

[8] J. Simon, Differentiation with respect to the domain in boundary value problems, Numer. Func. Anal. Optim. 2 (1980) 649-687.

[9] E. Zuazua, Finite-dimensional null controllability for the semilinear heat equation, J. Math. Pures Appl. (9) 76 no. 3 (1997) 237-264. 